\title{
Design and Modelling of Class EF Inverters for Wireless Power Transfer Applications
}

Ioannis Nikiforidis ${ }^{1}$, Juan M. Arteaga ${ }^{1}$, Christopher H. Kwan ${ }^{1}$, David C. Yates, Paul D. Mitcheson ${ }^{1}$

${ }^{1}$ Wireless Power Lab, Department of Electrical and Electronic Engineering, Imperial College London, London, United Kingdom

\begin{abstract}
Class EF inverters have been widely used recently as primary coil drivers for wireless power transfer applications since they achieve constant output current across a range of link coupling factor values. As the operating frequency that the inductive link is tuned at increases the traditional circuit design techniques that are based on first order calculations fail to represent the inverter behaviour accurately. In this paper, we present a novel method of modelling Class EF inverters that is based on state space representation of the circuit and thus providing the highest accuracy possible. Our method consists of a combination of analytical and numerical calculations in such manner that any parasitic component of the circuit, such as the nonlinear output capacitance of a power switch, can be included in the tuning process.
\end{abstract}

\section{Methodology outline}

In this paper we present a general method for designing Class EF inverters (Fig. 1) as primary coil drivers for wireless power transfer applications. The design algorithm consists of three main calculation parts as shown in Fig. 2. The process in the first part is a combination of the methods described in [1] and [2]. The second and third part are numerical and use the state space representation of the circuit with the inclusion of the effect of parasitic components. The output of the calculation process is the component values of the circuit and the voltage and current waveforms. The arithmetic calculations in the second part of the algorithm are a combination of Newton's method with gradient descent and the third part uses $4^{\text {th }}$ order Runge-Kutta and Bisection method specially designed and adapted for the convergence requirements of our use case. Our method achieves high operation efficiency compared to what can be found in the literature (Table 2).

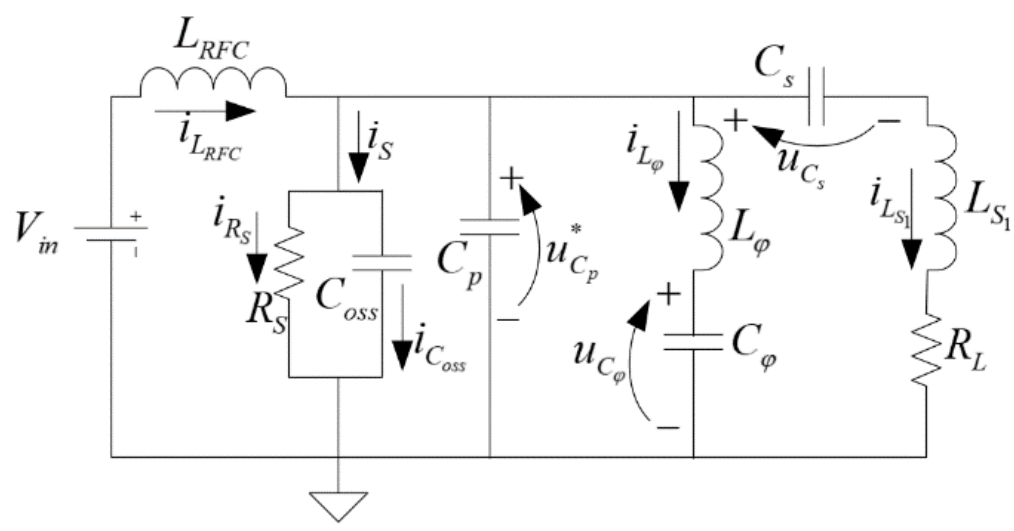

Fig. 1. EF inverter with the state space variables shown and the switch modelled as variable resistance in parallel to a voltage dependent capacitance.

\section{Technical details}

The tuning process of the inverter circuit begins by determining the output requirements of the IPT link, which are the output power $\left(P_{\mathrm{o}}\right)$, the total combined reflected load $\left(R_{L}\right)$ and the switching frequency $(f)$. Then, the desired values for the normalised tuning frequency of the $\varphi$ branch $\left(\tau_{\varphi}\right)$, duty cycle $(D)$, output to input current ratio $(\lambda)$ and primary coil inductance value $\left(L_{S I}\right)$ are chosen. Table 1 contains the numerical values of the input parameters which were taken from the setup described in [3]. 


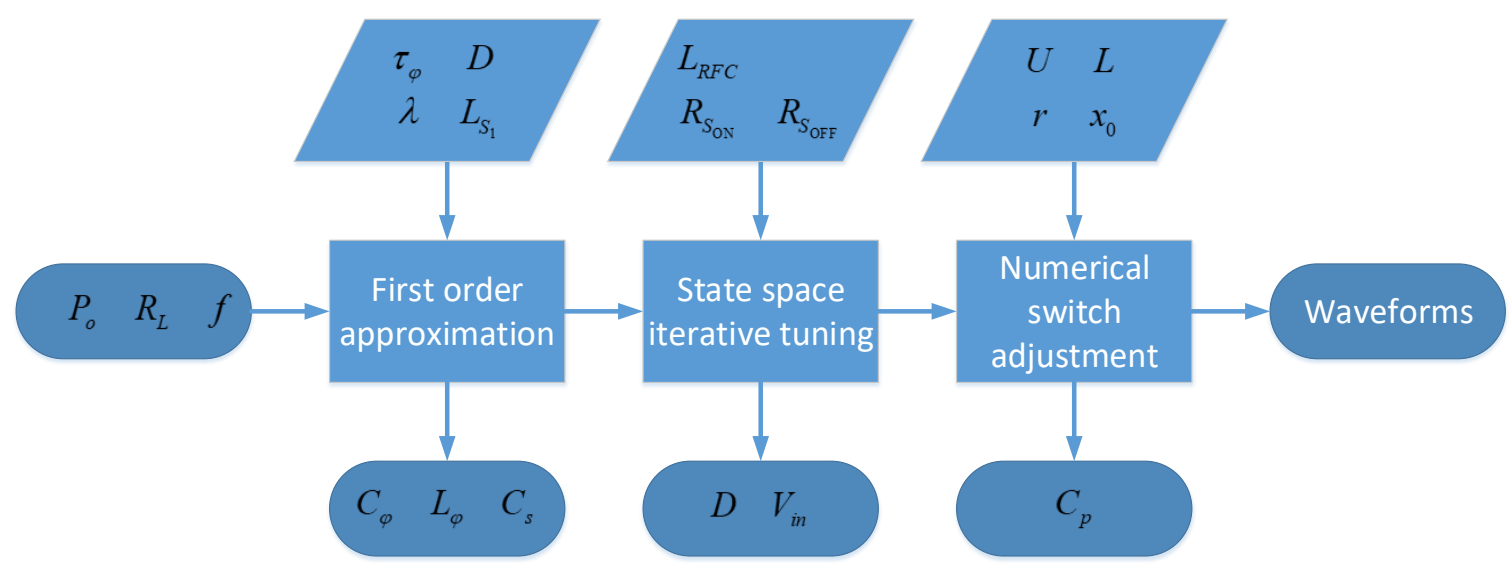

Fig. 2. EF inverter with the state space variables shown and the switch modelled as variable resistance in parallel to a voltage dependent capacitance.

\begin{tabular}{|c|r|l|c|r|l|}
\hline $\begin{array}{l}\text { Table 1. Input parameters with corresponding numerical values of the tuning } \\
\text { algorithm. }\end{array}$ \\
\hline \multicolumn{5}{|c|}{ Requirements } & \multicolumn{3}{c|}{ Conditions } \\
\hline Quantity & Value & Unit & Quantity & Value & Unit \\
\hline$P_{\mathrm{o}}$ & 150 & $\mathrm{~W}$ & $\tau_{\varphi}$ & 2 & - \\
\hline$R_{L}$ & 6 & $\Omega$ & $D$ & 30 & $\%$ \\
\hline$f$ & 13.56 & $\mathrm{MHz}$ & $\lambda$ & 5 & - \\
\hline
\end{tabular}

The boundary conditions of the EF branch originate from the continuity requirements during switching are described by (1). The residual output impedance was calculated numerically by applying Fourier analysis to the output signal.

$$
\left[\begin{array}{c}
i_{L_{\varphi}}\left(\theta_{\mathrm{OFF}}^{-}\right) \\
i_{L_{\varphi}}(\theta=0) \\
u_{C_{\varphi}}\left(\theta_{\mathrm{OFF}}^{-}\right) \\
u_{C_{\varphi}}(\theta=0)
\end{array}\right]=\left[\begin{array}{c}
i_{L_{\varphi}}\left(\theta_{\mathrm{OFF}}{ }^{+}\right) \\
i_{L_{\varphi}}\left(\theta_{\mathrm{ON}}\right) \\
u_{C_{\varphi}}\left(\theta_{\mathrm{OFF}}^{+}\right) \\
u_{C_{\varphi}}\left(\theta_{\mathrm{ON}}\right)
\end{array}\right]
$$

Then we use a a modified Newton-Raphson method to compute a set of components in the EF inverter that satisfies the required external operation conditions, which involve the behavior of the output current and the drain voltage and current. We use the sine fitting method described in [4] and the Jacobian is computed by the estimation technique in [5].

Finally, we compensate for the voltage dependent output capacitor of the switching device with a sigmoid approximation:

$$
C_{\text {oss }}^{S}\left(u_{D S}\right)=L+\frac{U-L}{1+e^{-r\left(u_{D S}-x_{0}\right)}}
$$

where $L, U, r$ and $x_{0}$ are fitting parameters. Since the circuit description in state space with the inclusion of $C_{o s s}$ is non-linear and there is no direct analytical solution, it is solved numerically by using the $4^{\text {th }}$ order Runge-Kutta iterative method. It is repeatedly solved during one period, until the boundary conditions are satisfied to the required tolerance.

We evaluated our model with an LTspice simulation. The circuit that was simulated is shown in Figure 3. The resulting waveforms are plotted in Figure 4. Our model has successfully incorporated the effect of the actual switching device and the two sets of waveforms agree highly. 


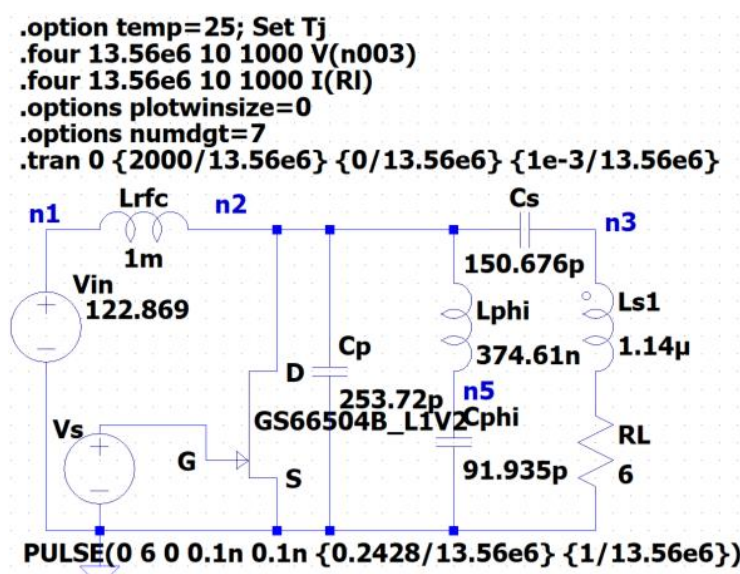

Fig. 3. LTspice circuit for evaluating the final EF model.
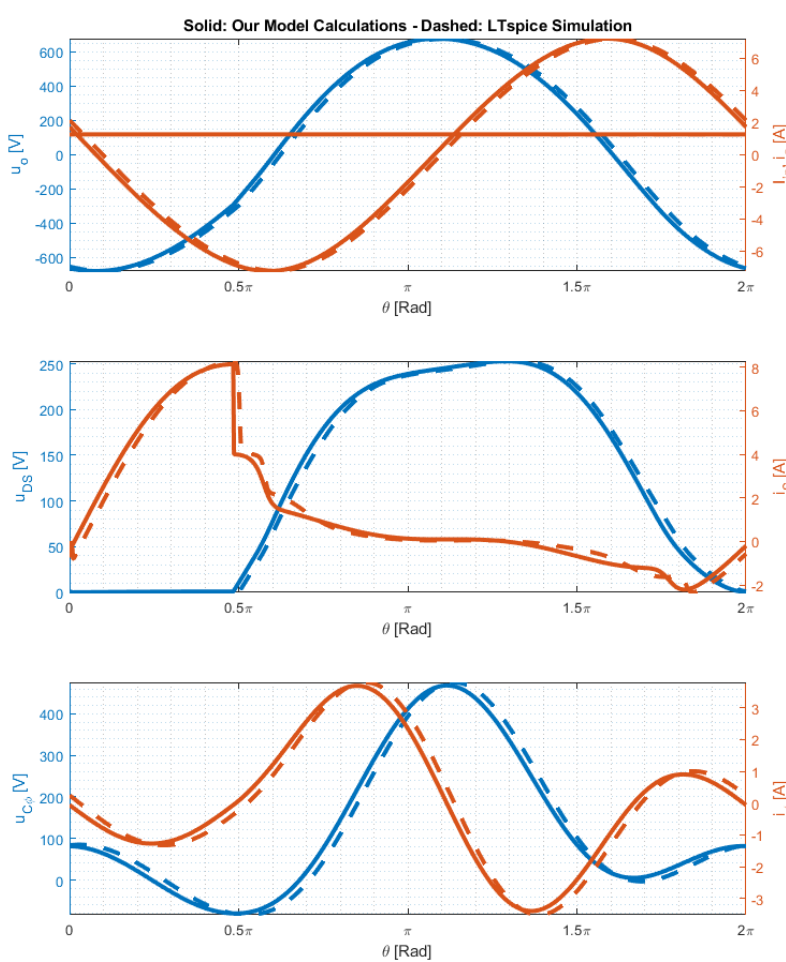

Fig. 4. State space solution with components from iterative tuning including the effect of $\mathrm{C}_{\text {oss }}$ (solid blue line) and corresponding LTspice simulation with the GS66504B of Gan Systems switching device model (dashed orange line).

\begin{tabular}{|c|c|c|c|}
\hline \multicolumn{4}{|c|}{$\begin{array}{l}\text { Table 2.Comparison between our design approach } \\
\text { (left) and the one described in [2] (right) for a } 150 \mathrm{~W} \\
\text { load. }\end{array}$} \\
\hline$I_{\text {out } D C}$ & 0.673 & 12.632 & $\mu \mathrm{A}$ \\
\hline$T H D_{i_{\text {ovt }}}$ & 1.195 & 7.597 & $\%$ \\
\hline$P_{\text {out }}$ & 152.811 & 142.044 & $\mathrm{~W}$ \\
\hline$\eta$ & 99.097 & 89.558 & $\%$ \\
\hline
\end{tabular}

\section{Summary}

In this paper we described a novel way of calculation for the electrical component values of Class EF inverters for wireless power transfer applications. Our method includes a variety of techniques to achieve the highest accuracy possible with the corresponding experimental setup, going beyond just the typical first order approximation. Comparison with LTspice simulation and the results from the existing literature confirm that the complexity that our approach introduces to the calculating process provides higher level of accuracy. 
Future work involves the description of the measurement method of the component values for these high frequency applications so that our theoretical results are as close as possible with the experimental setup. Possibly a calibration of the theoretical tuning method to the experimental data will be required. Our goal is to define a straight forward and automated inverter tuning method with minimum amount of manual adjustment needed.

\section{Acknowledgements}

The authors gratefully acknowledge the financial support from the European Union's Horizon 2020 research and innovation programme under the Marie Sklodowska-Curie Grant Agreement No. 722496 and EPSRC grant ref: EP/R004137/1 Converter Architectures.

\section{References}

[1] F. H. Raab, "Idealized Operation of the Class E tuned Power Amplifier", IEEE Transactions on Circuits and Systems, vol. 24, no. 12, Dec. 1977.

[2] S. Aldhaher, D. C. Yates and P. D. Mitcheson, "Modeling and Analysis of Class EF and Class E/F Inverters With Series-Tuned Resonant Networks", IEEE Transactions on Power Electronics, vol. 31, no. 5, May 2016.

[3] S. Aldhaher, D. C. Yates and P. D. Mitcheson, "Load-Independent E/EF Inverters and Rectifiers for MHz-Switching Applications", IEEE Transactions on Power Electronics, vol. 33, no. 10, Oct. 2018.

[4] P. Handel, "Evaluation of a Standardized Sine Wave Fit Algorithm".

[5] J. R. R. A. Martins, P. Sturdza and J. J. Alonso, "The Complex-Step Derivative Approximation", ACM Transactions on Mathematical Software, vol. 29, no. 3, pp. 245-262, Sep. 2003.

Corresponding author: Ioannis Nikiforidis, Imperial College London, South Kensington Campus, London, United Kingdom, SW7 2AZ, +44 7935 830331, i.nikiforidis@imperial.ac.uk. 